\title{
Unsafe abortion: why restricting abortive drugs only makes a bad situation worse
}

This Feature contains some inaccuracies in the numbering of its references (BMJ 2013;346:f3159, doi:10.1136/bmj.f3159). The reference at the end of paragraph 11 should have read reference 7 , rather than 6 . In paragraph 12 , the reference at the end of the first sentence should have been reference 8 (not 7). At the end of paragraph 17 the reference should have been reference 9 (not 8), and the reference at the end of the second sentence in paragraph 19 should have also been reference 9 (not 8).

Cite this as: BMJ 2013;346:14078

๑ BMJ Publishing Group Ltd 2013 